\title{
Construction and Validation of a Nomogram for Non-small Cell Lung Cancer Patients with Liver Metastases: A Population-Based Analysis
}

\section{Ruhan Zhao}

Affiliated Hospital of Chengde Medical University

Yunnan Dai

Affiliated Hospital of Chengde Medical University

\section{Xinyang Li}

Affiliated Hospital of Chengde Medical University

Cuimin Zhu ( $\nabla$ zzlk116@126.com)

Affiliated Hospital of Chengde Medical University

\section{Research Article}

Keywords: Non-small cell lung cancer, Liver metastases, Nomogram, SEER, Prognosis

Posted Date: July 28th, 2021

DOI: https://doi.org/10.21203/rs.3.rs-744039/v1

License: (9) This work is licensed under a Creative Commons Attribution 4.0 International License.

Read Full License

Version of Record: A version of this preprint was published at Scientific Reports on March 7th, 2022. See the published version at https://doi.org/10.1038/s41598-022-07978-8. 


\section{Abstract}

Background: Lung cancer is one of the most common malignancies in the United States, and the common metastatic sites in advanced non-small cell lung cancer (NSCLC) are bone, brain, adrenal gland, and liver, respectively, among which patients with liver metastases have the worst prognosis.

Methods: We retrospectively analyzed 1963 patients diagnosed with NSCLC combined with liver metastases between 2010 and 2015. Independent prognostic factors for patients with liver metastases from NSCLC were identified by univariate and multivariate Cox regression analysis. Based on this, we developed a nomogram model via R software and evaluated the performance and clinical utility of the model by calibration curve, receiver operating characteristic curves, and decision curve analysis (DCA).

Result: The independent prognostic factors for NSCLC patients with liver metastases included age, race, gender, grade, T stage, $\mathrm{N}$ stage, brain metastases, bone metastases, surgery, chemotherapy, and tumor size. The area under the curve predicting OS at 6,9 , and 12 months was $0.793,0.787$, and 0.784 in the training cohort, and $0.767,0.771$, and 0.773 in the validation cohort, respectively. Calibration curves of the nomogram showed high agreement between the outcomes predicted by the nomogram and the actual observed outcomes, and the DCA further demonstrated the value of the clinical application of the nomogram.

Conclusion: By analyzing the Surveillance, Epidemiology, and End Results database, we established and verified a prognostic nomogram for NSCLC patients with liver metastases, to personalize the prognosis of patients. At the same time, the prognostic nomogram has a satisfactory accuracy and the results are a guide for the development of patient treatment plans.

\section{Introduction}

Lung cancer is currently the most common and deadliest malignancy in the world, and the number of new diagnoses and deaths of lung cancer patients in the United States is expected to be 235,760 and 131,880 , respectively, in 2021, according to cancer data published in the United States[1]. Lung cancer can be divided into two categories: non-small cell lung cancer (NSCLC) and small cell lung cancer, and NSCLC account for approximately $85 \%$ of lung cancer cases, due to clinical manifestations and signs are not obvious, and about $40 \%-55 \%$ of these patients are advanced at the time of diagnosis[2]. The common sites of metastasis in advanced NSCLC are bone, brain, adrenal gland, and liver[3]. For advanced patients presenting with metastases, their median survival is 8-12 months, while in patients with metastases at common sites, the median OS is only 3 months once liver metastases are diagnosed $[4,5]$. It has also been demonstrated that liver metastasis is one of the independent poor prognostic factors for patients with NSCLC, and therefore patients with liver metastases from NSCLC have the worst prognosis[6]. TNM staging is now widely accepted as a tool to predict patient prognosis[7]. However, the patient's age, gender, marital status, pathological features, distant metastatic sites and numbers, and treatment 
methods all have an impact on their prognosis[8]. Therefore, it is still difficult to accurately predict the prognosis of metastatic lung cancer using TNM staging.

Compared with brain metastases and bone metastases, the prognosis of patients with liver metastases from non-small cell lung cancer has been less studied, and there is no effective model to predict the prognosis of NSCLC patients with liver metastases. The nomogram is a simple statistical tool that can meet our needs for a comprehensive clinical model. Nomograms enable rapid calculations as well as higher accuracy and easier to understand prognosis compared to traditional TNM staging to assist physicians in clinical decision making[9]. Therefore, through this study, we aimed to integrate the clinical characteristics of patients in the Surveillance, Epidemiology, and End Results database (SEER) database between 2010 and 2015 and quantify the impact of each risk to create a nomogram, which can make the results of the prediction model more readable through visual graphs and can more accurately determine the prognosis of NSCLC patients with liver metastases, helping clinicians to be able to better develop treatment strategies.

\section{Methods}

\section{Patient selection}

We screened patients diagnosed with NSCLC combined with liver metastases between 2010 and 2015 in the SEER database. Because patient information in the SEER database is publicly available and free of charge, institutional review board approval was not required for this study. Inclusion criteria were: (1) patients aged $\geq 18$ years, (2) patients whose only primary site tumor was pathologically diagnosed as NSCLC, (3) those with complete information, including race, primary site, grade, marital status, surgery, chemotherapy, radiation therapy, and insurance status. We finally screened 1963 NSCLC patients with liver metastases for inclusion in this study.

\section{Variable definitions}

We extracted factors from the patient data that might be associated with prognosis, including age, gender, race, laterality, primary site, histological type, grade, T stage, $\mathrm{N}$ stage, surgery, radiotherapy, chemotherapy, bone metastases, brain metastases, lung metastases, marital status, and insurance status. Age was changed from a continuous variable to a categorical variable by $\mathrm{X}$-tile software and divided into $<55,55-66$ and $>66$. Similarly, the tumor size is divided into $<42,42-71$ and $>71$. The tumor site was divided into the main bronchus, upper lobe, middle lobe, lower lobe, and overlapping sites based on anatomy. According to the 8th edition of the American Joint Committee on cancer guidelines, $T$ was divided into $\mathrm{T} 1, \mathrm{~T} 2, \mathrm{~T} 3$, and $\mathrm{T} 4$, and similarly, $\mathrm{N}$ was divided into N1, N2, and N3. The primary endpoint of this study was overall survival (OS), defined as the time interval from the date of diagnosis to the date of patient death.

\section{Statistical Analysis}


We randomly divided patients into a training group $(n=1375)$ and a validation group $(n=588)$ in a ratio of 7:3. The variables associated with prognosis in NSCLC with liver metastases were identified by univariate Cox regression analysis of prognosis-related indicators. Subsequently, variables with $P$ values $<0.05$ in the univariate Cox regression analysis were subjected to multivariate Cox regression analysis to obtain independent prognostic factors for NSCLC with liver metastases, and multiple predictors were integrated to assign different scores using the degree of contribution of different factors to OS. Finally, a nomogram model for predicting OS in NSCLC patients with liver metastases was established by converting the scores to a functional relationship with OS using $\mathrm{R}$ software. The model performance is divided into two main aspects, discrimination and calibration, which we have validated in the training and validation groups, respectively. We used calibration curves to measure the agreement between predicted and actual outcomes. The discriminant of the model was measured by calculating the receiver operating characteristic curves (ROC) curves area under the curve (AUC), which took values in the range of 0.5-1.0. Finally, the clinical application value of the model was evaluated by decision curve analysis (DCA). The random grouping, nomogram, calibration curves, ROC, and DCA were composed by R language software (version 4.0.3). Bilateral $P$ values $<0.05$ were considered statistically significant.

\section{Results}

\section{Baseline characteristics of NSCLC patients with liver metastases}

A total of 199,564 patients with confirmed NSCLC were collected in the SEER database between 2010 and 2015, and 1963 eligible cases were finally obtained, of which 1375 were randomly assigned to the training group and 588 were randomly assigned to the validation group. Table 1 summarizes the demographic and clinical characteristics of the NSCLC patients with liver metastases. In the training group, 733 (53.3\%) patients were $>66$ years old, 1073 (78\%) were white, $804(58.4 \%)$ were male, and 1330 $(96.7 \%)$ patients had insurance. For tumor characteristics, $54.6 \%$ of them were adenocarcinoma, $59.2 \%$ were upper lobe, $68.4 \%$ were histologic grade III, and $50.2 \%$ were N2. Among the modalities of follow-up treatment received, the majority (97\%) of patients did not receive surgical treatment, $55.7 \%$ received chemotherapy, and $42.9 \%$ received radiation therapy.

\section{Development and validation of a prognostic nomogram for NSCLC patients with liver metastases}

After univariate Cox regression analysis, a total of 14 factors were significantly associated with the prognosis of NSCLC patients with liver metastases $(P<0.05)$, including age, race, gender, histological type, grade, T stage, $\mathrm{N}$ stage, brain metastases, bone metastases, lung metastases, surgery, chemotherapy, insurance status, and tumor size (Table 2). These factors were then subjected to multivariate Cox regression analysis, and finally, 11 factors were identified as independent prognostic factors for NSCLC with liver metastases including age, race, gender, grade, T stage, $\mathrm{N}$ stage, brain metastases, bone metastases, surgery, chemotherapy, and tumor size. We established a prognostic nomogram for NSCLC patients with liver metastases based on the independent prognostic factors selected in the multivariate Cox regression analysis (Figure 1), from which it is clear that the $T$ stage has the greatest impact on the 
prognosis of NSCLC patients with liver metastases, followed by tumor size. The area under the curve of the clinical prognostic model predicting overall survival at 6,9 , and 12 months was $0.793,0.787$, and 0.784 in the training group and $0.767,0.771$, and 0.773 in the validation group, respectively (Figure 2 ). In addition, we further compared the difference of AUC value between nomogram and all independent prognostic factors and the results showed that the AUC value of the nomogram was higher than the AUC of all independent factors at 6,9 , and 12 months, both in the training group and the validation group (Figure 3). The calibration curves are shown in Figure 4 . The curves are all close to 45 degrees, indicating that the prognostic nomogram has a good calibration performance. Also, the DCA showed that the prognostic nomogram has strong clinical utility (Figure 5). Based on the cut-off values of risk scores, patients were classified into high risk, medium risk, and low risk groups. By depicting the Kaplan-Meier survival curve, we can find that as the risk increases, the prognosis of the patients will become worse (Figure 6).

\section{Discussion}

The incidence of liver metastases in lung cancer ranges $2.9-4.1 \%$ and is $20-30 \%$ in patients with NSCLC, which makes the prognosis worse[10]. The risk of death in patients with NSCLC in the presence of liver metastases is 1.53-2.41 times higher than that of distant metastases from other sites[8, 11]. However, there is no relevant model that can personalize the prognosis of patients with liver metastases from NSCLC. Therefore, we created a prognostic nomogram for NSCLC patients with liver metastases by analyzing the basic information of patients in the SEER database and achieved a more precise to determine the prognosis of patients by corresponding each variable to the nomogram graph. The study confirmed that race, age, gender, grade, $\mathrm{T}$ stage, $\mathrm{N}$ stage, tumor size, presence of distant metastases from remaining sites (e.g., brain, bone, etc.), surgery, and chemotherapy were all associated with prognosis.

The results of this study showed that females had a better prognosis compared to males in NSCLC patients with liver metastases, which may be related to the stimulating effect of androgens on the growth of lung cancer[12]. The 8th edition of the AJCC guidelines states that regardless of the patient's T or N stage, as long as distant metastases are present, their stage is stage IV. A study has shown that $\mathrm{T}$ and $\mathrm{N}$ stages cannot be ignored even in stage IV patients and that $\mathrm{T}$ and $\mathrm{N}$ stages indicate to a certain extent the malignancy of tumor cells and determine the treatment of NSCLC patients[13]. The study showed that the more advanced the $\mathrm{T}$ and $\mathrm{N}$ stages and the larger the tumor size, the worse the prognosis of the patients, probably because as the tumor size increases, the sensitivity of the tumor cells to treatments such as radiotherapy decreases[14]. Similarly, the higher the histological grade of the patient, the more aggressive the tumor tissue is, and accordingly the shorter the survival of the patients[15]. In patients with NSCLC, adenocarcinoma, compared to squamous carcinoma and other histological types, is more likely to develop liver metastases[16]. However, the histologic type is not one of the poor prognostic factors for NSCLC patients with liver metastases, which may be due to different chemotherapy regimens for patients with different histologic types. Among patients with single-site metastases from NSCLC, liver metastases have a relatively shorter median survival compared to brain or bone metastases. Patients with multisite metastases had a worse prognosis than single-site metastases, and among patients with multisite 
metastases, OS was reduced by 1 month or more in patients with liver metastases than in patients without liver metastases[17].

The current standard treatment for NSCLC patients with liver metastases is systemic treatment, while for liver metastases, local treatments such as surgery, radiotherapy, radiofrequency ablation, and interventional therapy are available [18]. Hepatectomy may be a treatment option when the patient's liver metastasis is single lesions[19]. Sun et al. demonstrated that for NSCLC patients with only three metastases or less and single metastases, surgery can improve OS, as well as reduce the tumor burden of patients and reduce or eliminate tumor-induced complications, which can improve patient prognosis to some extent[20,21]. Although surgery significantly improves the prognosis, the vast majority of patients are lost to surgery at diagnosis, and only $3 \%$ of patients in our study underwent surgical treatment[22]. According to the previous opinion, patients with liver metastases from NSCLC have a relatively low response rate to chemotherapy, which may be since some patients with liver metastases have liver dysfunction and cannot tolerate chemotherapy[23]. A study by katsunori et al. showed a median survival of 6 months for patients receiving chemotherapy, which improved overall survival by 4 months compared to patients receiving only symptomatic supportive therapy[16]. Similarly, our study confirms that chemotherapy improves the prognosis of patients with liver metastases from NSCLC. Consistent with previous studies radiotherapy did not show an advantage in multivariate Cox regression analysis, suggesting that radiotherapy does not improve OS in patients with liver metastases from NSCLC, but radiotherapy can still be used as one of the palliative treatments to relieve pain, reduce complications and improve patients' quality of life[7].

There are some limitations to our study. First, targeted therapies (TKIs) and immunotherapy are currently available for some populations of non-small cell lung cancer, but specific information on these treatments is not available in the SEER database, so we were unable to analyze whether these two treatments modalities could improve patient prognosis. Secondly, the study was a retrospective analysis, and details of the treatment modalities such as the surgical approach, chemotherapy regimen, and the site and dose of radiotherapy were not available. Third, the SEER database only collects data from the United States, and further research is needed to determine whether this nomogram is generalizable to other countries and ethnic groups.

\section{Conclusions}

In brief, we established a prognostic nomogram for NSCLC patients with liver metastases by analyzing the basic information in the SEER database, which contains 11 variables including race, age, gender, grade, T stage, $\mathrm{N}$ stage, tumor size, bone metastases, brain metastases, surgery, and chemotherapy. Clinicians can use the prognostic nomogram to accurately assess the OS of patients, identify high-risk patients, and provide a reference for optimizing treatment plans. 


\section{Declarations}

\section{Ethics approval and consent to participate}

Not applicable, as the study is based on a publicly available dataset.

\section{Consent for publication}

Not applicable.

\section{Competing interests}

The authors declare that they have no competing interests.

\section{Funding}

We received no external funding for this study.

\section{Author Contributions}

$\mathrm{RH} Z$ and $\mathrm{CM} \mathrm{Z}$ conceived of and designed the study. RH Z and YN D performed literature search. YN D, $\mathrm{XY} \mathrm{L}$ generated the figures and tables. $\mathrm{RH} Z$ analyzed the data. $\mathrm{RH} \mathrm{Z}$ wrote the manuscript and $\mathrm{CM} \mathrm{Z}$ critically reviewed the manuscript. $\mathrm{RH} Z$ and $\mathrm{CM} \mathrm{Z}$ supervised the research.

\section{Acknowledgements}

None

\section{References}

1. Siegel RL, Miller KD, Fuchs HE, Jemal A: Cancer Statistics, 2021. CA: a cancer journal for clinicians 2021, 71(1):7-33.

2. D Morgensztern, SH Ng, F Gao, R Govindan: Trends in stage distribution for patients with nonsmall cell lung cancer: a National Cancer Database survey. Journal of thoracic oncology : official publication of the International Association for the Study of Lung Cancer 2010, 5(1):29-33.

3. Hakoda H, Sekine Y, Ichimura H, Ueda K, Aoki S, Mishima H, Sako A, Kobayashi K, Sakata A, Sato Y: Hepatectomy for rapidly growing solitary liver metastasis from non-small cell lung cancer: a case report. Surgical case reports 2019, 5(1):71.

4. Ponz-Sarvisé M, Nguewa PA, Pajares MJ, Agorreta J, Lozano MD, Redrado M, Pio R, Behrens C, Wistuba II, García-Franco CE et al: Inhibitor of differentiation-1 as a novel prognostic factor in NSCLC patients with adenocarcinoma histology and its potential contribution to therapy resistance. Clinical 
cancer research : an official journal of the American Association for Cancer Research 2011, 17(12):41554166.

5. Ren Y, Dai C, Zheng H, Zhou F, She Y, Jiang G, Fei K, Yang P, Xie D, Chen C: Prognostic effect of liver metastasis in lung cancer patients with distant metastasis. Oncotarget 2016, 7(33):53245-53253.

6. Corrao G, Marvaso G, Ferrara R, Lo Russo G, Gugliandolo SG, Piperno G, Spaggiari L, De Marinis F, Orecchia R, Garassino MC et al: Stereotatic radiotherapy in metastatic non-small cell lung cancer: Combining immunotherapy and radiotherapy with a focus on liver metastases. Lung cancer (Amsterdam, Netherlands) 2020, 142:70-79.

7. J Deng, Z Ren, J Wen, B Wang, X Hou, Z Xue, X Chu: Construction of a nomogram predicting the overall survival of patients with distantly metastatic non-small-cell lung cancer. Cancer management and research 2018, 10:6143-6156.

8. M Riihimäki, A Hemminki, M Fallah, H Thomsen, K Sundquist, J Sundquist, K Hemminki: Metastatic sites and survival in lung cancer. Lung cancer (Amsterdam, Netherlands) 2014, 86(1):78-84.

9. VP Balachandran, M Gonen, JJ Smith, RP DeMatteo: Nomograms in oncology: more than meets the eye. The Lancet Oncology 2015, 16(4):e173-180.

10. Stenbygaard LE, Sørensen JB, Larsen H, Dombernowsky P: Metastatic pattern in non-resectable non-small cell lung cancer. Acta oncologica (Stockholm, Sweden) 1999, 38(8):993-998.

11. Tamura T, Kurishima K, Nakazawa K, Kagohashi K, Ishikawa H, Satoh H, Hizawa N: Specific organ metastases and survival in metastatic non-small-cell lung cancer. Molecular and clinical oncology 2015, 3(1):217-221.

12. Salmerón $D$, Chirlaque MD, Isabel Izarzugaza M, Sánchez MJ, Marcos-Gragera R, Ardanaz E, Galceran J, Mateos A, Navarro C: Lung cancer prognosis in Spain: the role of histology, age and sex. Respiratory medicine 2012, 106(9):1301-1308.

13. Zhu J, Wang W, Xu S, Jia C, Zhang Q, Xia Y, Wang W, Wen M, Wang X, Wang H et al: Evaluation of the Effect of Lymph Node Status on the Survival of Non-Small Cell Lung Cancer Patients With Brain Metastases: Applications of a Novel Grade Prognostic Assessment Score Model Involving N Stage. Frontiers in oncology 2020, 10:563700.

14. Ball D, Mitchell A, Giroux D, Rami-Porta R: Effect of tumor size on prognosis in patients treated with radical radiotherapy or chemoradiotherapy for non-small cell lung cancer. An analysis of the staging project database of the International Association for the Study of Lung Cancer. Journal of thoracic oncology : official publication of the International Association for the Study of Lung Cancer 2013, 8(3):315-321. 
15. Jiang T, Cheng R, Zhang G, Su C, Zhao C, Li X, Zhang J, Wu F, Chen X, Gao G et al: Characterization of Liver Metastasis and Its Effect on Targeted Therapy in EGFR-mutant NSCLC: A Multicenter Study. Clinical lung cancer 2017, 18(6):631-639.e632.

16. Kagohashi K, Satoh H, Ishikawa H, Ohtsuka M, Sekizawa K: Liver metastasis at the time of initial diagnosis of lung cancer. Medical oncology (Northwood, London, England) 2003, 20(1):25-28.

17. Lu YJ, Yang Y, Yuan YH, Wang WJ, Cui MT, Tang HY, Duan WM: A novel nomogram based on SEER database for the prediction of liver metastasis in patients with small-cell lung cancer. Annals of palliative medicine 2020, 9(5):3123-3137.

18. Jiang T, Chu Q, Wang H, Zhou F, Gao G, Chen X, Li X, Zhao C, Xu Q, Li W et al: EGFR-TKIs plus local therapy demonstrated survival benefit than EGFR-TKIs alone in EGFR-mutant NSCLC patients with oligometastatic or oligoprogressive liver metastases. International journal of cancer 2019, 144(10):26052612.

19. Ileana E, Greillier L, Moutardier V, Barlesi F: Surgical resection of liver non-small cell lung cancer metastasis: a dual weapon? Lung cancer (Amsterdam, Netherlands) 2010, 70(2):221-222.

20. Sun Z, Sui X, Yang F, Wang J: Effects of primary tumor resection on the survival of patients with stage IV extrathoracic metastatic non-small cell lung cancer: A population-based study. Lung cancer (Amsterdam, Netherlands) 2019, 129:98-106.

21. Abdel-Rahman O: Outcomes of Surgery as Part of the Management of Metastatic Non-Small-Cell Lung Cancer: A Surveillance, Epidemiology and End Results Database Analysis. Cancer investigation 2018, 36(4):238-245.

22. $\mathrm{Li}$ J, Zhu H, Sun L, Xu W, Wang X: Prognostic value of site-specific metastases in lung cancer: A population based study. Journal of Cancer 2019, 10(14):3079-3086.

23. Wu W, Liao H, Ye W, Li X, Zhang J, Bu J: Fatty liver is a risk factor for liver metastasis in Chinese patients with non-small cell lung cancer. PeerJ 2019, 7:e6612.

\section{Tables}




\begin{tabular}{|c|c|c|c|c|}
\hline Variables & Training cohort & & Validation cohort & \\
\hline & $N=1375$ & & $N=588$ & \\
\hline & $\mathrm{n}$ & प & $\mathrm{n}$ & प \\
\hline \multicolumn{5}{|l|}{ Age } \\
\hline$<55$ & 162 & 11.7 & 89 & 15.1 \\
\hline $55-66$ & 480 & 35.0 & 200 & 34.0 \\
\hline$\varangle 66$ & 733 & 53.3 & 299 & 50.9 \\
\hline$₫ 66$ & 733 & & & \\
\hline \multicolumn{5}{|l|}{ Race } \\
\hline Black & 144 & 10.5 & 57 & 9.7 \\
\hline Other & 158 & 11.5 & 59 & 10.0 \\
\hline White & 1073 & 78.0 & 472 & 80.3 \\
\hline \multicolumn{5}{|l|}{ Sex } \\
\hline Male & 804 & 58.5 & 338 & 57.5 \\
\hline Female & 571 & 41.5 & 250 & 42.5 \\
\hline \multicolumn{5}{|l|}{ Primary site } \\
\hline Lower lobe & 413 & 30.1 & 192 & 32.7 \\
\hline Main bronchus & 63 & 4.6 & 26 & 4.4 \\
\hline Middle lobe & 68 & 4.9 & 31 & 5.3 \\
\hline Overlapping lesion of lung & 17 & 1.2 & 7 & 1.2 \\
\hline Upper lobe & 814 & 59.2 & 332 & 56.4 \\
\hline \multicolumn{5}{|l|}{ Laterality } \\
\hline Left & 613 & 44.6 & 213 & 36.2 \\
\hline Right & 762 & 55.4 & 375 & 63.8 \\
\hline \multicolumn{5}{|l|}{ Histological types } \\
\hline Adenocarcinoma & 752 & 54.7 & 322 & 54.8 \\
\hline Other & 245 & 17.8 & 109 & 18.5 \\
\hline Squamous cell carcinoma & 378 & 27.5 & 157 & 26.7 \\
\hline
\end{tabular}




\begin{tabular}{|c|c|c|c|c|}
\hline \multicolumn{5}{|c|}{ Grade } \\
\hline I & 65 & 4.7 & 26 & 4.4 \\
\hline II & 335 & 24.4 & 144 & 24.5 \\
\hline III & 941 & 68.4 & 401 & 68.2 \\
\hline IV & 34 & 2.5 & 17 & 2.9 \\
\hline \multicolumn{5}{|c|}{ T stage } \\
\hline $\mathrm{T} 1$ & 112 & 8.1 & 51 & 8.7 \\
\hline $\mathrm{T} 2$ & 388 & 28.2 & 156 & 26.5 \\
\hline T3 & 395 & 28.7 & 176 & 29.9 \\
\hline $\mathrm{T} 4$ & 480 & 35.0 & 205 & 34.9 \\
\hline \multicolumn{5}{|c|}{ N stage } \\
\hline No & 272 & 19.8 & 115 & 19.6 \\
\hline N1 & 122 & 8.9 & 42 & 7.1 \\
\hline N2 & 691 & 50.2 & 293 & 49.8 \\
\hline N3 & 290 & 21.1 & 138 & 23.5 \\
\hline \multicolumn{5}{|c|}{ Surgery } \\
\hline No & 41 & 3.0 & 568 & 96.6 \\
\hline Yes & 1334 & 97.0 & 20 & 3.4 \\
\hline \multicolumn{5}{|c|}{ Chemotherapy } \\
\hline No & 608 & 44.2 & 247 & 42.0 \\
\hline Yes & 767 & 55.8 & 341 & 58.0 \\
\hline \multicolumn{5}{|c|}{ radiotherapy } \\
\hline No & 785 & 57.1 & 335 & 57.0 \\
\hline Yes & 590 & 42.9 & 253 & 43.0 \\
\hline \multicolumn{5}{|c|}{ Bone metastasis } \\
\hline No & 667 & 48.5 & 278 & 47.3 \\
\hline Yes & 708 & 51.5 & 310 & 52.7 \\
\hline \multicolumn{5}{|c|}{ Brain metastasis } \\
\hline No & 1004 & 73.0 & 442 & 75.2 \\
\hline
\end{tabular}




\begin{tabular}{|llccc|}
\hline Yes & 371 & 27.0 & 146 & 24.8 \\
\hline Lung metastasis & & & & \\
Yes & 881 & 64.1 & 384 & 65.3 \\
\hline Tumor size & 494 & 35.9 & 204 & 34.7 \\
$\square 42$ & & & & \\
$42-71$ & 521 & 37.9 & 227 & 38.6 \\
\hline 71 & 555 & 40.4 & 219 & 37.2 \\
\hline Insurance status & 299 & 21.7 & 142 & 24.2 \\
\hline No & & & & 2.0 \\
\hline Yes & 45 & 3.3 & 12 & 98.0 \\
\hline Marital & 1330 & 96.7 & 576 & 45.7 \\
\hline No & & & & 54.3 \\
\hline Yes & 608 & 44.2 & 269 & \\
\hline
\end{tabular}


Table 2 Univariate and multivariate Cox regression analysis in NSCLC patients with liver metastases Univariate Cox analysis Multivariate Cox analysis $\begin{array}{llllll}\mathrm{HR} & 95 \% \mathrm{Cl} & \mathrm{P} & \mathrm{HR} & 95 \% \mathrm{Cl} & \mathrm{P}\end{array}$

\section{Age}

$<55$

1

1

$55-66$

$\begin{array}{llll}1.212 & 1.006 & 1.460 & 0.043\end{array}$

1.151

$0.954 \quad 1.390$

0.143

$₫ 66$

$\begin{array}{llll}1.383 & 1.157 & 1.653 & 0.000\end{array}$

1.316

1.09

$\begin{array}{ll}1.579 & 0.003\end{array}$

\section{Race}

Black 1

1

Other

0.724

0.572

$0.917 \quad 0.007$

0.803

0.632

$\begin{array}{ll}1.022 & 0.074\end{array}$

White

$\begin{array}{llll}1.030 & 0.862 & 1.231 & 0.746\end{array}$

1.178

0.981

$\begin{array}{ll}1.414 & 0.079\end{array}$

Sex

Female

Male

1

1

Primary site

Lower lobe

1

Main bronchus

$\begin{array}{llll}1.086 & 0.826 & 1.428 & 0.555\end{array}$

Middle lobe

$\begin{array}{llll}0.873 & 0.664 & 1.148 & 0.330\end{array}$

Overlapping lesion of

lung

Upper lobe

1.289

1.153

1.441

0.000

1.235

1.103

$1.384 \quad 0.000$

\section{Laterality}

Left

1

Right

$\begin{array}{llll}0.954 & 0.855 & 1.065 & 0.400\end{array}$

\section{Histological types}

Adenocarcinoma

Other

$\begin{array}{llll}1.624 & 0.984 & 2.680 & 0.058\end{array}$

1.023

0.905

1.156

0.718

Squamous cell carcinoma

\section{Grade}




\begin{tabular}{|c|c|c|c|c|c|c|c|c|}
\hline I & 1 & & & & 1 & & & \\
\hline II & 1.160 & 0.871 & 1.544 & 0.310 & 1.003 & 0.747 & 1.347 & 0.985 \\
\hline III & 1.673 & 1.273 & 2.198 & 0.000 & 1.362 & 1.026 & 1.807 & 0.032 \\
\hline IV & 1.708 & 1.104 & 2.643 & 0.016 & 1.333 & 0.853 & 2.084 & 0.207 \\
\hline T sta & & & & & & & & \\
\hline T1 & 1 & & & & 1 & & & \\
\hline $\mathrm{T} 2$ & 1.460 & 1.168 & 1.825 & 0.001 & 1.317 & 1.035 & 1.676 & 0.025 \\
\hline T3 & 1.735 & 1.389 & 2.167 & 0.000 & 1.367 & 1.067 & 1.752 & 0.014 \\
\hline T4 & 1.725 & 1.386 & 2.148 & 0.000 & 1.449 & 1.137 & 1.847 & 0.003 \\
\hline $\mathrm{N}$ sta & & & & & & & & \\
\hline No & 1 & & & & 1 & & & \\
\hline N1 & 0.933 & 0.744 & 1.168 & 0.544 & 0.877 & 0.696 & 1.103 & 0.262 \\
\hline N2 & 1.166 & 1.007 & 1.349 & 0.040 & 1.221 & 1.046 & 1.424 & 0.011 \\
\hline N3 & 1.262 & 1.064 & 1.496 & 0.007 & 1.336 & 1.115 & 1.602 & 0.002 \\
\hline Surg & & & & & & & & \\
\hline No & 1 & & & & 1 & & & \\
\hline Yes & 0.399 & 0.275 & 0.580 & 0.000 & 0.484 & 0.330 & 0.711 & 0.000 \\
\hline Chen & & & & & & & & \\
\hline No & 1 & & & & 1 & & & \\
\hline Yes & 0.407 & 0.364 & 0.456 & 0.000 & 0.365 & 0.324 & 0.411 & 0.000 \\
\hline Radi & & & & & & & & \\
\hline No & 1 & & & & & & & \\
\hline Yes & 0.990 & 0.887 & 1.105 & 0.857 & & & & \\
\hline Bone & & & & & & & & \\
\hline No & 1 & & & & 1 & & & \\
\hline Yes & 1.226 & 1.098 & 1.368 & 0.000 & 1.323 & 1.179 & 1.484 & 0.000 \\
\hline Brain & & & & & & & & \\
\hline No & 1 & & & & 1 & & & \\
\hline Yes & 1.156 & 1.024 & 1.307 & 0.020 & 1.232 & 1.086 & 1.398 & 0.001 \\
\hline
\end{tabular}




\begin{tabular}{|c|c|c|c|c|c|c|c|c|}
\hline \multicolumn{9}{|c|}{ Lung metastasis } \\
\hline No & 1 & & & & & & & \\
\hline Yes & 1.198 & 1.069 & 1.341 & 0.002 & & & & \\
\hline \multicolumn{9}{|c|}{ Tumor size } \\
\hline$\nabla 42$ & 1 & & & & 1 & & & \\
\hline $42-71$ & 1.207 & 1.065 & 1.368 & 0.003 & 1.078 & 0.944 & 1.232 & 0.267 \\
\hline$\otimes 71$ & 1.715 & 1.480 & 1.987 & 0.000 & 1.410 & 1.195 & 1.664 & 0.000 \\
\hline \multicolumn{9}{|c|}{ Insurance status } \\
\hline No & 1 & & & & & & & \\
\hline Yes & 0.693 & 0.511 & 0.940 & 0.019 & & & & \\
\hline \multicolumn{9}{|c|}{ Marital } \\
\hline No & 1 & & & & & & & \\
\hline Yes & 0.901 & 0.807 & 1.006 & 0.064 & & & & \\
\hline
\end{tabular}

Figures 


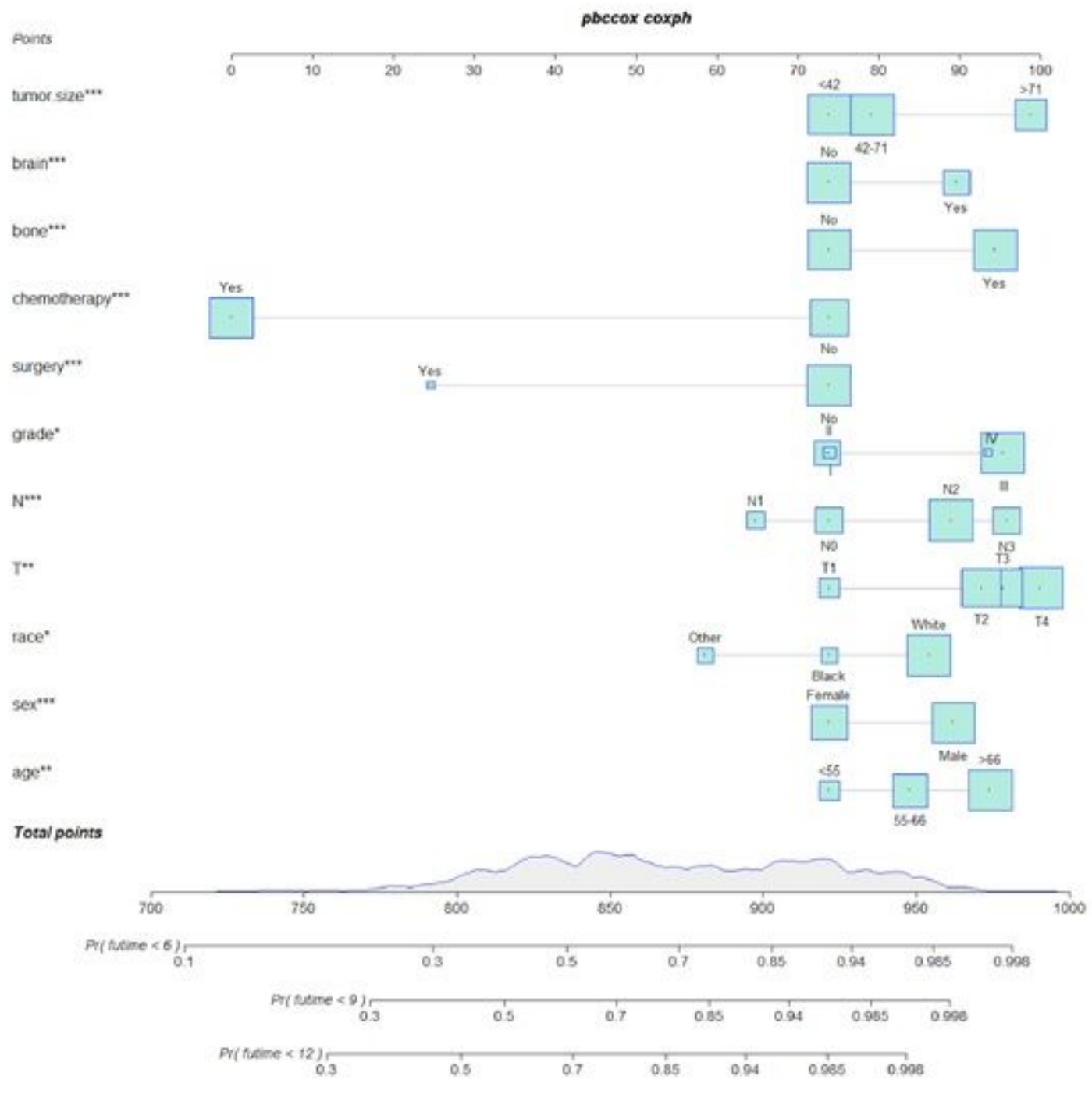

Figure 1

A prognostic nomogram for NSCLC patients with liver metastases.
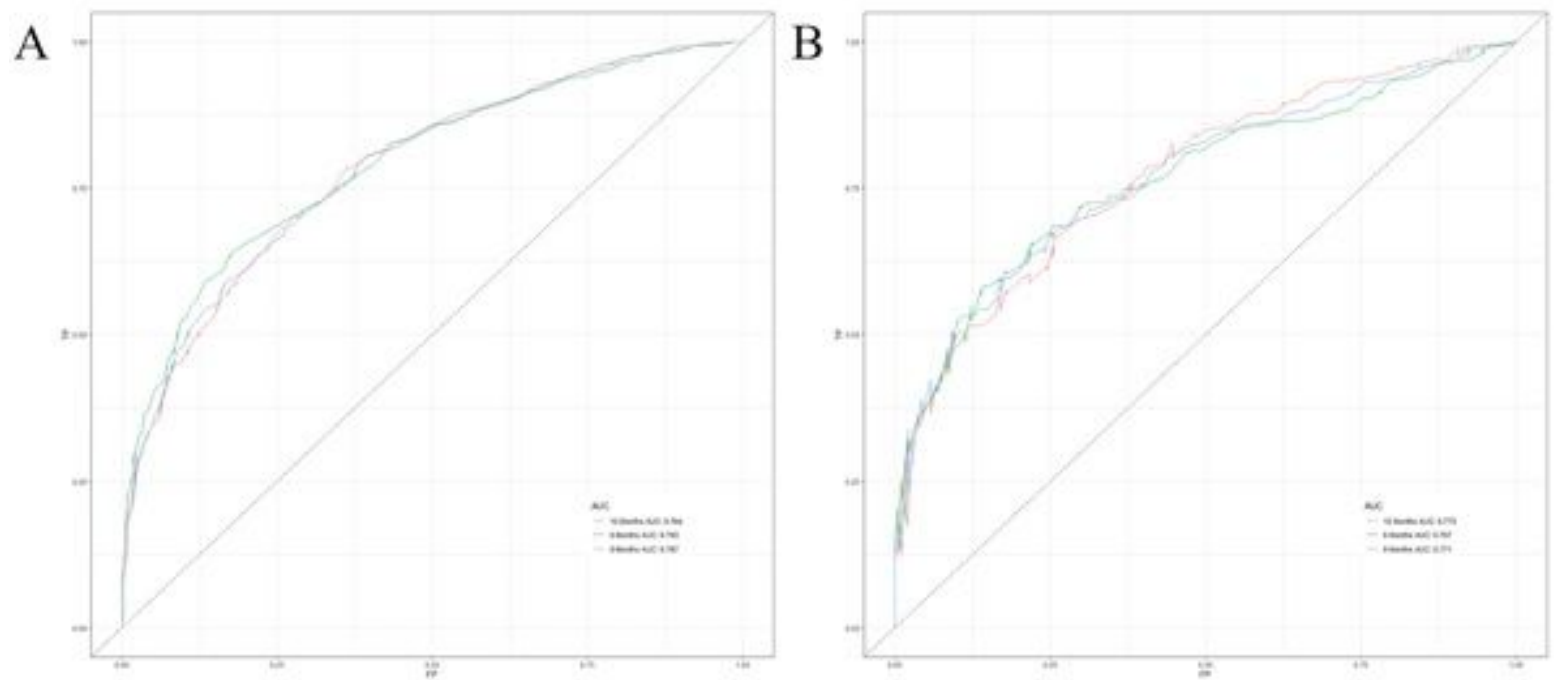

Figure 2 
ROC curves for NSCLC patients with liver metastases. (A) ROC curves of 6-, 9-, and 12-months in the training group, (B) ROC curves of 6-, 9-, and 12-months in the validation group. ROC: Receiver operating characteristic.

A
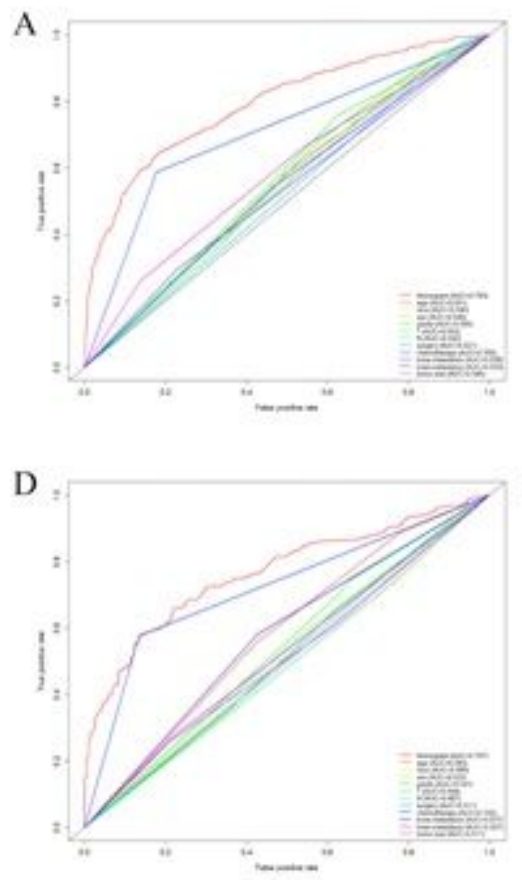

B

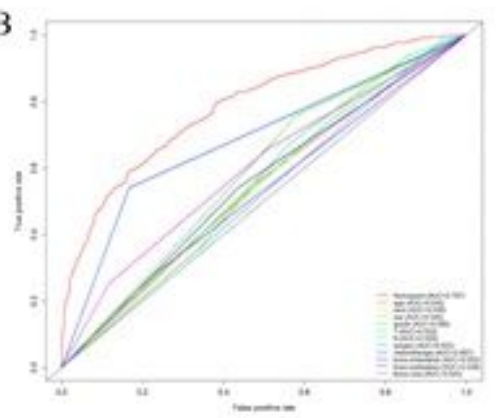

E

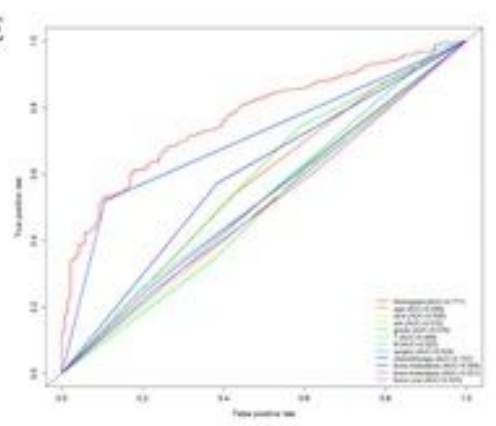

$\mathrm{C}$

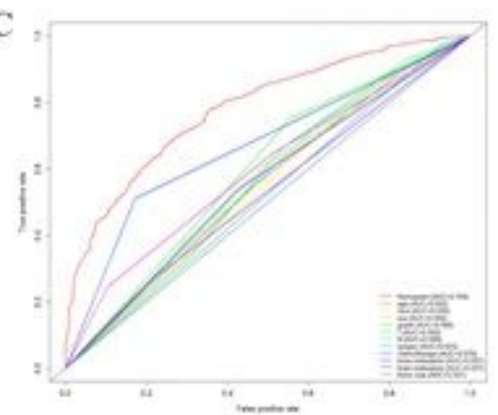

$\mathrm{F}$

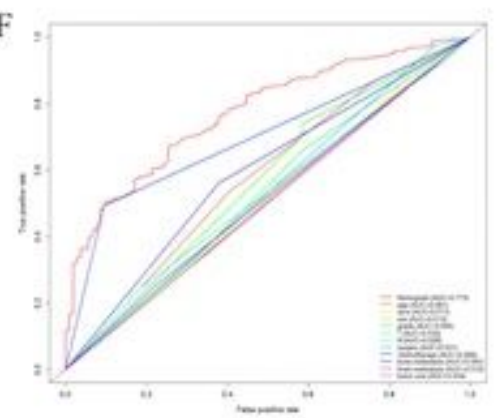

\section{Figure 3}

The ROC curves of nomogram and all independent predictors at 6- (A), 9-(B), and 12-months (C) in the training group and at 6-(D), 9- (E), and 12-months (F) in the validation group.

A

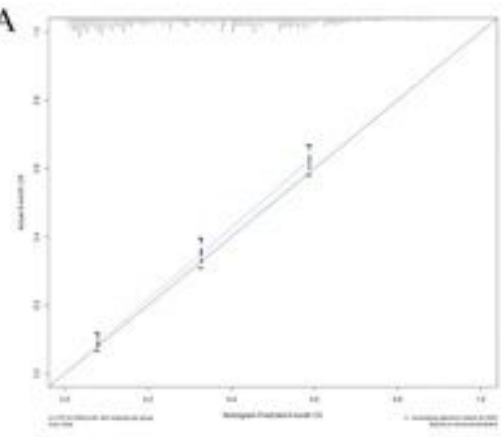

D

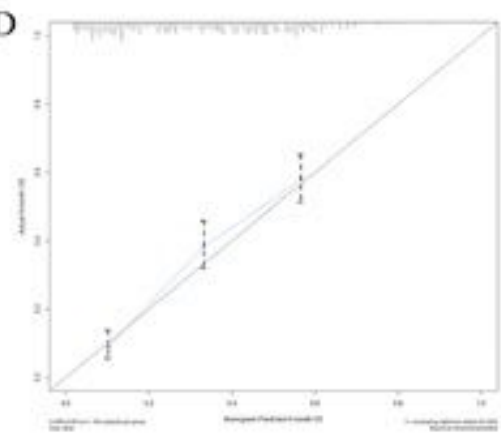

B

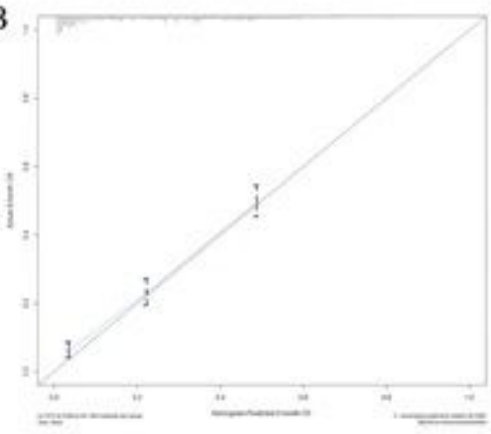

E

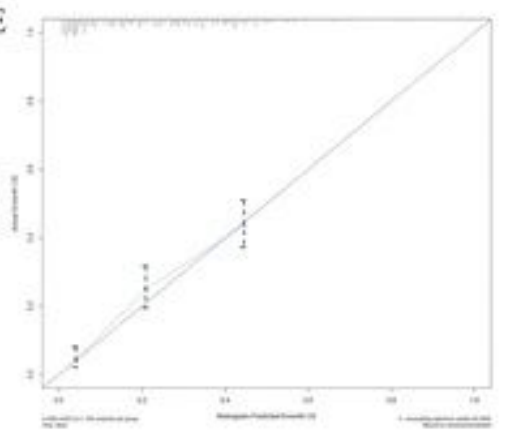

C
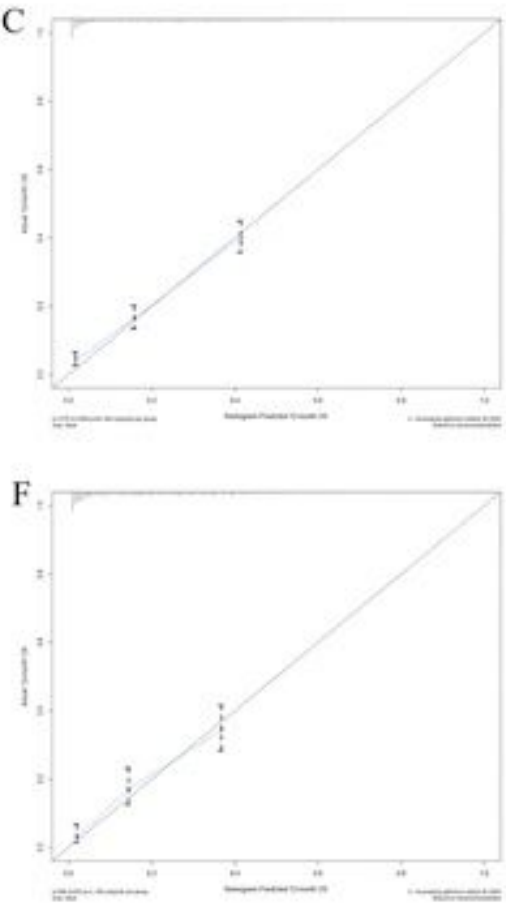

Figure 4 
The calibration curves of the nomogram for the 6-, 9-, and 12-months OS prediction of the training group $(A-C)$ and validation group $(D-F)$. The $x$ axis represents the nomogram- predicted survival rates, whereas the $y$ axis represents the actual survival rates.
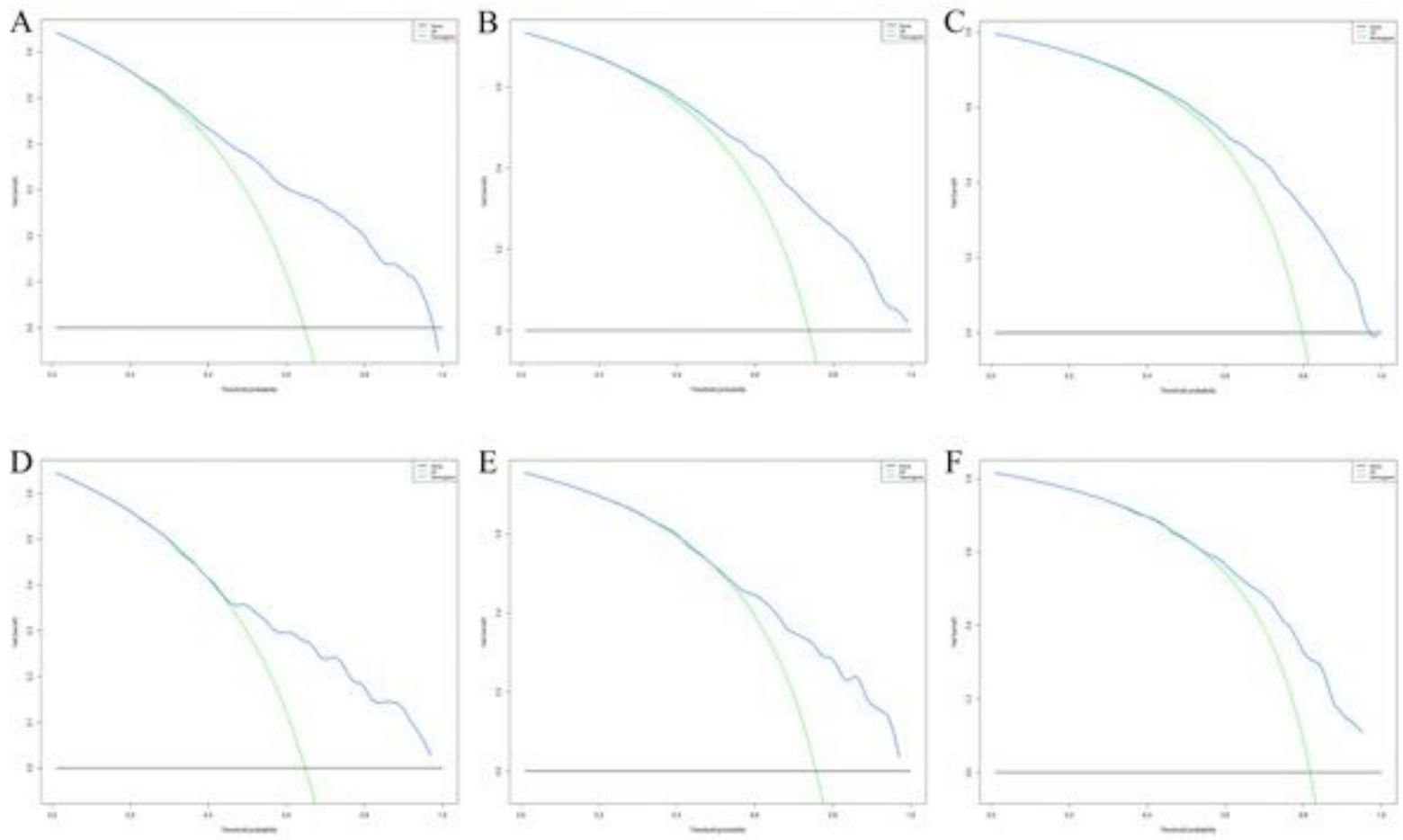

Figure 5

DCA of the nomogram for the survival prediction of NSCLC patients with liver metastases. (A) 6-months survival benefit in the training group. (B) 9-months survival benefit in the training group. (C) 12-months survival benefit in the training group. (D) 6-months survival benefit in the validation group. (E) 9-months survival benefit in the validation group. (F) 12-months survival benefit in the validation group.
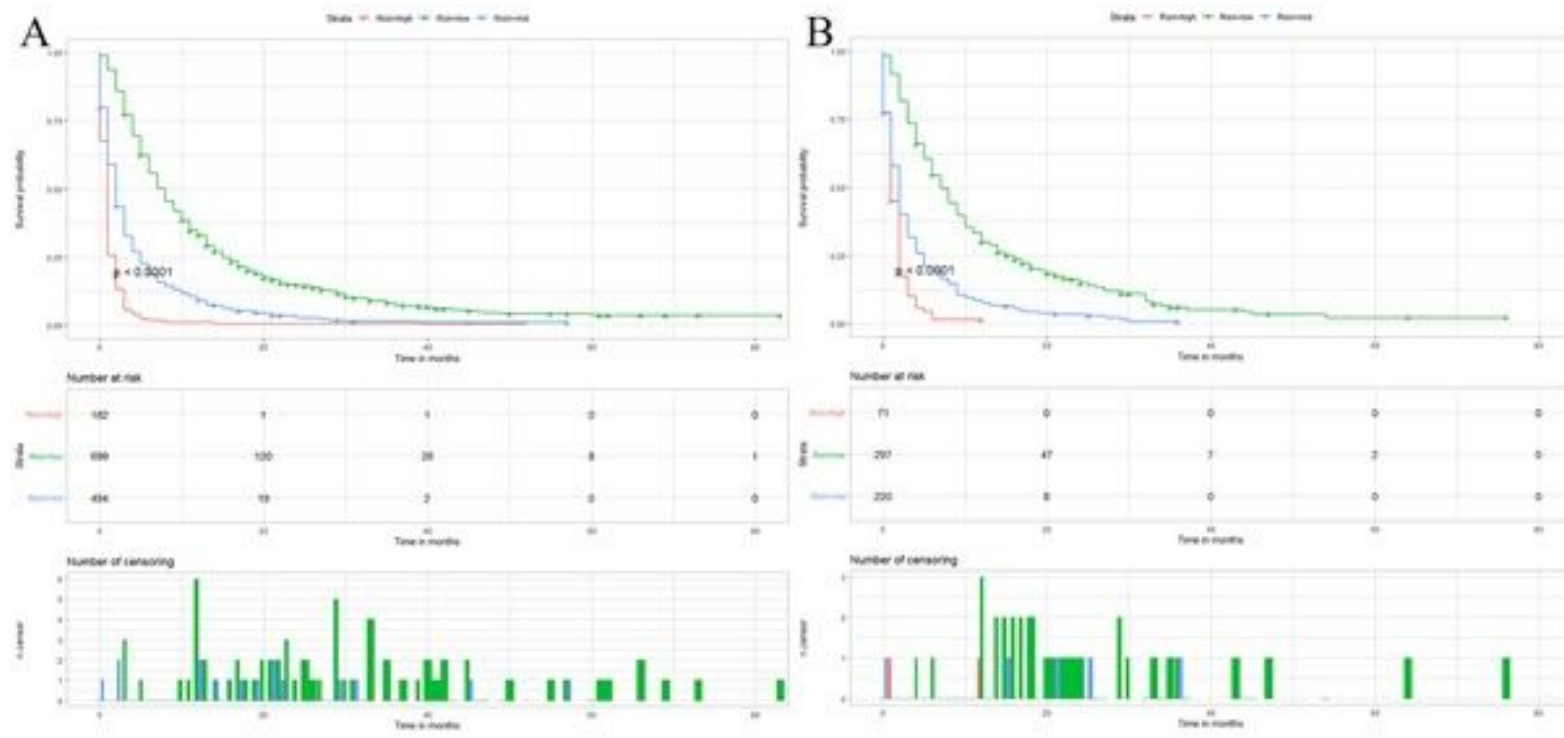

Figure 6 
Kaplan-Meier survival analysis of the signature for both the training group and the validation group. Patients with a higher risk score demonstrated a worse OS than those with a low risk score in the training group (A) and validation group (B), which suggests the strong predictive ability for NSCLC patients with liver metastases survival outcome. 